\title{
From High School to Medical School: the Importance of Community in Education
}

\author{
Lars Osterberg • Jeff Gilbert • Rachel Lotan
}

\author{
Published online: 3 July 2014 \\ (C) International Association of Medical Science Educators (Outside the U.S.A.) 2014
}

\section{Learning Communities}

Learning is often curtailed in large anonymous settings where academic and social pressures, unreasonable workload, and highstake examinations are constant. Whether in high school or professional school, many students often feel at a loss and isolated, becoming disengaged and depressed in such unsupportive learning environments $[1,2]$. Creating a supportive setting where students can interact productively, manage the requirements of the curriculum, and develop a sense of belonging is imperative. Learning communities (LCs) are learning environments created within larger settings where students and faculty members of these communities can establish close and sustained professional and personal connections, common goals, and most importantly, can learn together and from each other.

First described by McMillan and Chavis in 1986, learning communities have members who share common values and beliefs; they actively engage in learning together and from each other [3]. These smaller groups of students maintain cohesion and develop a sustained relationship with one or a few faculty members in longitudinal relationships. Within these smaller student groups and longitudinal faculty relationships, the foundation of a learning community is created.

Learning communities have become more prevalent over the past 20 years in higher education [4]. In secondary schools, learning communities are introduced to increase student engagement and support teachers in providing quality instruction (http:// www.betterhighschools.org/map/learning.asp). In this paper, we

\section{Osterberg $(\bowtie)$}

Stanford University School of Medicine and Veterans Affairs

Hospital, Palo Alto, CA, USA

e-mail: larso@stanford.edu

J. Gilbert

Hillsdale High School Principal, San Mateo, CA, USA

R. Lotan

Stanford University, Palo Alto, CA, USA describe the rationale and the goals of learning communities in a high school and in a medical school using the examples of Hillsdale High School and Stanford Medical School, respectively, both on the San Francisco Peninsula, CA, USA.

\section{Conceptual Framework}

Because learning is a social activity [5, 6], sustained professional and personal relationships with instructors and peers helps contribute to effective learning. When students have opportunities to discuss, grapple with dilemmas, and solve problems, they deepen their understanding and develop the discourse of their particular discipline. Setting the stage for such productive interactions requires structural changes in the learning environment from large, anonymous settings to smaller, closer, and intimate surroundings. Thus, students often find comfort in a learning environment created by subdividing large student groups into smaller ones where students and faculty can get to know one another and form longitudinal connections. A sense of community among students and faculty, which is a key aspect provided by LCs, has also proven to be an important component in preventing burnout in students and faculty $[7,8]$.

Social learning theory states that learning takes place within a social context and is facilitated through modeling and observational learning [9]. A combination of environmental and psychological factors influences student behavior, and faculty role models become critical in the education of young students [10]. Therefore, choosing respected faculty advisors that care about their students, and can make a longitudinal commitment to them, is essential for a supportive and effective LC.

\section{Small Learning Communities in High School: the Case of Hillsdale High}

The transition of adolescents from middle school to high school is often marked by a sense of anxiety and confusion. 
High school completes a shift away from the personalized, nurturing environment of grade school, often replacing it with a largely incoherent system - more students, unsustained relationships, disparate grading and behavior expectations, and higher stakes with less individualized supports. Some traditional high schools, built up and calcified over decades, have resulted in an impersonal culture that can prioritize the needs of the institution over those of the learner.

Hillsdale High School, located in San Mateo, CA, in Silicon Valley, is one of a number of schools that are challenging the traditional high school structures by creating learning communities within the larger comprehensive school. Hillsdale is integrating the best features of these small schools, while maintaining the advantages of larger high schools - orchestras, badminton teams, and a broader array of course offerings. Guiding this work is a core set of cornerstone values (equity, personalization, rigor, and shared decision making) that drive decision making and focus the school on a common vision. The structures that have been evolved from the cornerstones have created a coherent learning environment not always seen in traditional schools.

At the heart of Hillsdale's learning community design is the organization of the lower division (9th/10th grades) and upper division (11th/12th grades) each into three semi-autonomous LCs. In the ninth grade, for example, 324 students are randomly and heterogeneously sorted into three cohorts, with each group of 108 students assigned to a set of four teachers, including english, history, science, and math. The students then stay with those teachers for 2 years, while taking two or three other classes outside of the LC. The LC teachers' only assignment is to teach those 108 students and then serve as the advisor for a quarter of the cohort (each faculty advises 27 students). This advisory model allows each teacher in the LC to meet with his or her students everyday, to serve as counselor, on-site parent, disciplinarian, and shoulder to cry on. Each LC also has a dedicated counselor and administrator to serve as a support network for the team, but the heart of the model is the long-term and consistent relationships that form between the students and teachers in a given LC.

Learning communities have intentionally shifted Hillsdale's culture in the recognition that both academic and personal development is dependent upon the learning environment. For the adults, working in intensive teams with a consistent cohort of students, the cultural shift has been away from isolation toward collaboration. Teacher teams are provided common preparation periods and meet several hours each week to discuss student needs, curriculum, instruction, and assessment. Grading systems, behavior expectations, and curriculum units are co-constructed so that teachers know that their work is being reinforced across the classrooms. This has facilitated a shift away from a content focus to a student focus, so that the teacher teams think about the unique needs of each learner first, and the course content as a tool to impact growth rather than an end in itself. The interdependence that has resulted from teacher collaboration has also resulted in a shift from an accountability mindset to one of responsibility, where teachers work hard and effectively out of a commitment to the team and their students rather than a fear of reprimand.

For the students, LCs have shifted the experience from one of anonymity to one of sustained relationships, where trust exists between students and between students and teachers. This sense of belonging results in a willingness to take risks, fail, and try again with support. The world in a LC is vastly simpler for a student than it is in a traditional high school, with fewer systems and personal whims to understand and fewer adjustments to make, a critical issue for students in transition. Coherence in the structural model shifts the relationship between student and teacher, from one of expert-novice to one of a partnership, so that the student can more easily coconstruct knowledge rather than make sense of a mountain of information that is simply dumped on her.

Through the changes described above, LCs have allowed Hillsdale to increase the academic performance and fulfillment by its students. Hillsdale's performance on CA's Academic Performance Index (API), which measures results on state tests and sets 800 as the goal for schools, has risen from 662 before LCs to 818 in 2013, with 214 point gains for Latino students, 273 point gains for low socio-economic students, and substantial increases for every measured subgroup. In addition to improved student performance, the school is also supportive, positive, and united. The chair of a recent accreditation team, who had spent most of a week at Hillsdale, described it as the happiest high school in the world. Faculty who care about and see the best in every student, have contributed to the improved performance and support that has resulted in these positive outcomes of performance and satisfaction of the students.

\section{Learning Communities in Medical School: the Case of the Stanford School of Medicine Learning Community}

Similar to students transitioning to high school, medical students in large traditional medical schools often experience an impersonal culture as certain academic medical centers prioritize the needs of the institution over the needs of the learner [11]. In addition, medical students have the added stress of educational costs and the workload they face. While these financial and workload pressures are significant, more concerning is the erosion of well-being during medical training. The prevalence of depression, anxiety, burnout, and substance abuse significantly exceed those of their aged matched cohorts with anxiety and depression ranging from $20-56 \%$ and burnout as high as $53 \%$ in some studies $[12,13]$. The need for a supportive learning environment and resources to promote and maintain wellness in students during their training and into their careers as physicians is evident. 
Fig. 1 E4C students $(N=33)$ scored significantly higher $(p<0.02)$ than non-E4C students $(N=38)$ on all domains of the Clinical Performance Exam (CPX)

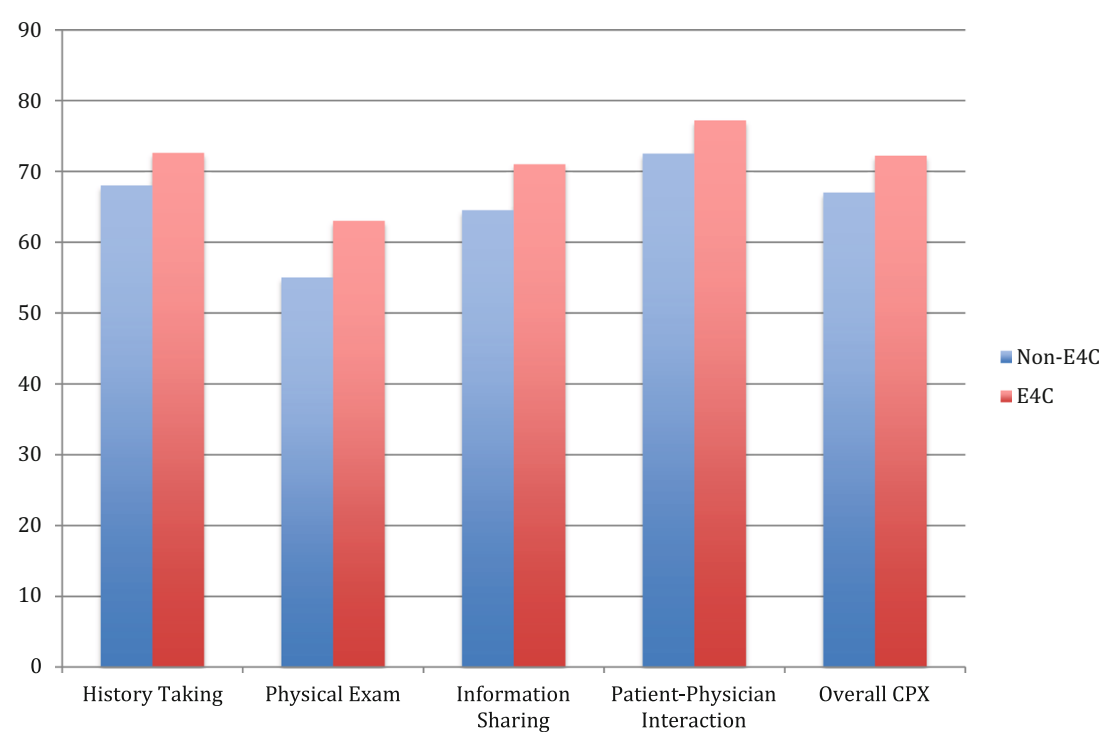

The Stanford Medical School LC, known as Educators for CARE (Compassion, Advocacy, Responsibility and Empathy) started in 2008 . The program was primarily developed to support curriculum delivery, and includes mentoring and general support for students. Five to six students from each class are grouped together with one of 17 faculty members in the Educators for CARE (E4C) learning communities. In 2008, the program had 16 faculty members, but due to the increase in Stanford Medical School class size in 2013, one more faculty mentor was added. Balancing gender, undergraduate school training, and whether the student is also a Ph.D. candidate, among other details, helps to create diversity within groups. Students continue in their LC throughout medical school working in small groups to learn their clinical skills and develop a longitudinal relationship with their faculty mentor. As the relationships grow in these small groups, students find comfort in learning in a safe environment and in sharing their experiences in caring for patients. The E4C program survey of students in 2010 demonstrated student comfort with sharing intense clinical experiences rated at 4.53 on a $1-5$ Likert scale. During the clinical rotations, students meet in small groups with their faculty and share for the first time many critical incidents they experience on the wards such as death and dying, breaking bad news, or the delivery of their first baby. The safety of the long-term relationship with the faculty allows for open expression of feelings and critical reflection on clinical experiences.

It was a great safe place to share concerns about rotations and ways to cope.- third year Stanford Medical Student, from E4C Program Survey in 2010

Since instituting the E4C program, there have been improvements in a number of outcomes attributable to the
Fig. 2 Empathy scores (Jefferson Scale of Physician Empathy) of Stanford students did not significantly decline during the clinical years of training as the scores in the students of the schools of Boston 2007 and Jefferson 2002 and 2004 $\left(p<0.02^{*}\right)$

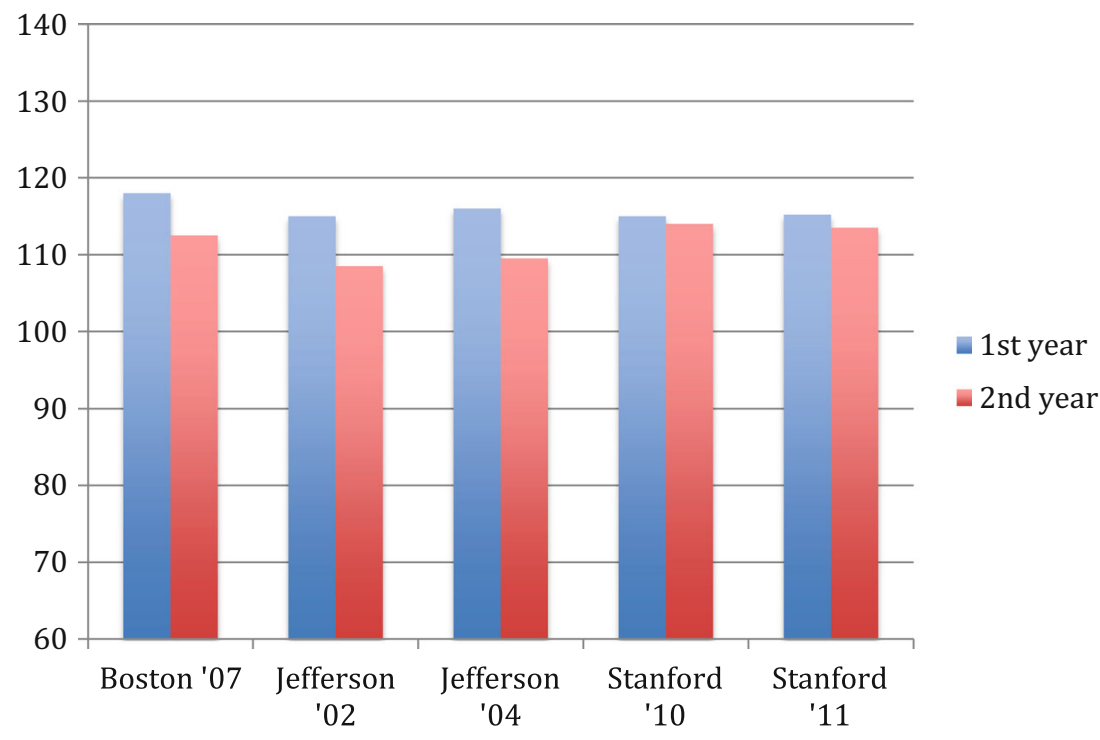


program. Students who have been mentored by faculty in the E4C program have significantly better clinical performance examination (CPX) scores compared to students who were not mentored by E4C faculty (see Fig. 1). Note that we had a number of students taking the CPX who did not have E4C faculty, since they entered medical school before the program was instituted and were not assigned E4C mentors. The vital promotion of student wellness is also supported through the mentorship and support provided by the E4C faculty [14]. A well-known phenomenon seen in medical schools is the drop in empathy in medical students as they transition through their first clinical year of training [15]. In measuring the empathy scores of medical students at Stanford, there has not been a statistically significant decline in empathy during clinical training as seen at other schools (see Fig. 2). In addition, the E4C faculty reported an overall improvement in job satisfaction and sense of community as a result of joining the $\mathrm{E} 4 \mathrm{C}$ program. Our E4C faculty survey in September 2012 revealed 10 of 13 faculty indicated increased job satisfaction, and 10 of 13 faculty surveyed indicated an increased sense of belonging at Stanford since starting the E4C program (note, 3 faculty did not complete the survey and only 16 faculty were in the program at that time).

\section{Conclusion}

Students in large schools, whether in traditional high schools, universities, or medical schools, experience similar pressures as they navigate the impersonal environments of their institutions. Integrating LCs within the learning environment to deliver curriculum creates coherence for students and provides a mechanism for support and caring through longitudinal relationships with faculty mentors. The content focus of large traditional institutions is replaced with a learner-centered focus of teaching and a responsibility mindset of faculty toward their students. As the learning community faculty work together to deliver curriculum, a learning community of faculty develops that creates more consistency in curriculum delivery and further elevates the quality of teaching by all faculty.

Learning communities have the potential to improve the learning environment in high schools, colleges, and medical schools through longitudinal role modeling by respected faculty, with student-centered learning that is refined by small group interactions. In this environment, students are more comfortable taking risks with known faculty and can apply and better grasp the extent and limits of their knowledge, pushing them to higher-level questions [16]. Curriculum delivery within the comfort of caring and known faculty improves learning performance and persistence of students [17]. Whether a student is experiencing the impersonal high-stress environment of high school, college, or medical training, all students can benefit from the supportive structure that learning communities provide.

\section{References}

1. Briere FN, Pascal S, Dupere V, Janosz M. School environment and adolescent depressive symptoms: a multilevel longitudinal study. Pediatrics. 2013;131(3):e702-8.

2. Dyrbye LN, Thomas MR, Harper W, et al. The learning environment and medical student burnout: a multicentre study. Med Educ. 2009;43(3):274-82.

3. Chavis DM, Hogge JH, McMillan DW, Wandersman A. Sense of community through Brunswick's lens: a first look. J Commun Psychol. 1986;14(1):24-40.

4. Love AG. The Growth and Current State of Learning Communities in Higher Education. New Directions for Teaching and Learning. 2012:5-18

5. Vygotsky LG. Mind in society: the development of higher psychological processes. Cambridge, Massachusetts: Harvard University Press; 1978.

6. Dewey J. Experience and education. Toronto, Canada: CollierMacMillan Canada Ltd.; 1938.

7. Leiter MP, Maslach C. Six areas of work life: a model of the organizational context of burnout. J Health Hum Serv Adm. 1999;21(4):472-89.

8. Shanafelt TD. Enhancing meaning in work: a prescription for preventing physician burnout and promoting patient-centered care. JAMA. 2009;302(12):1338-40.

9. Bahn D. Social learning theory: its application in the context of nurse education. Nurse Educ Today. 2001;21(2):110-7.

10. Maudsley RF. Role models and the learning environment: essential elements in effective medical education. Acad Med. 2001;76(5):432-4.

11. Ludmerer KM. Learner-centered medical education. N Engl J Med. 2004;351(12):1163-4.

12. Dyrbye LN, Massie Jr FS, Eacker A, et al. Relationship between burnout and professional conduct and attitudes among US medical students. JAMA. 2010;304(11):1173-80.

13. Dyrbye LN, Thomas MR, Shanafelt TD. Systematic review of depression, anxiety, and other indicators of psychological distress among U.S. and Canadian medical students. Acad Med. 2006;81(4):354-73.

14. Adams PJ, Basaviah P, Osterberg LG. Medical student wellness: an essential role for mentors. Med Sci Educ. 2011;21(4):282-384.

15. Hojat M, Mangione S, Nasca TJ, et al. An empirical study of decline in empathy in medical school. Med Educ. 2004;38(9):934- 41.

16. Irby DM, Wilkerson L. Educational innovations in academic medicine and environmental trends. J Gen Intern Med. 2003;18(5):370-6.

17. Brown AL. Transforming schools into communities of thinking and learning about serious matters. Am Psychol. 1997;52(4):399-413. 